\title{
Classification of Transient Myoelectric Signals for the Control of Multi-Grasp Hand Prostheses
}

\author{
Gunter Kanitz, Christian Cipriani, and Benoni B. Edin
}

(C) 2018 IEEE. Personal use of this material is permitted. Permission from IEEE must be obtained for all other uses, in any current or future media, including reprinting/republishing this material for advertising or promotional purposes, creating new collective works, for resale or redistribution to servers or lists, or reuse of any copyrighted component of this work in other works. The DOI associated with this publication is 10.1109/TNSRE.2018.2861465. The published version is available at the following

link: https://ieeexplore.ieee.org/document/8423679 


\title{
Classification of transient myoelectric signals for the control of multi-grasp hand prostheses
}

\author{
Gunter Kanitz, Christian Cipriani, Senior Member, IEEE, and Benoni B. Edin
}

\begin{abstract}
Understanding the neurophysiological signals underlying voluntary motor control and decoding them for controlling limb prostheses is one of the major challenges in applied neuroscience and rehabilitation engineering. While pattern recognition of continuous myoelectric (EMG) signals is arguably the most investigated approach for hand prosthesis control, its underlying assumption is poorly supported, i.e., that repeated muscular contractions produce consistent patterns of steady-state EMGs. In fact, it still remain to be shown that pattern recognition-based controllers allow natural control over multiple grasps in a hand prosthesis outside well-controlled laboratory settings. Here we propose an approach that relies on decoding the intended grasp from forearm EMG recordings associated with the onset of muscle contraction as opposed to the steadystate signals. Eight unimpaired individuals and two hand amputees performed four grasping movements with a variety of arm postures while EMG recordings subsequently processed to mimic signals picked up by conventional myoelectric sensors were obtained from their forearms and residual limbs, respectively. Off-line data analyses demonstrated the feasibility of the approach also with respect to the limb position effect. The sampling frequency and length of the classified EMG window that off-line resulted in optimal performance were applied to a controller of a research prosthesis worn by one hand amputee and proved functional in real-time when operated under realistic working conditions.
\end{abstract}

Index Terms- Myoelectric control, pattern recognition, onset of muscle contraction, prosthetic hand, transient control.

\section{INTRODUCTION}

$\mathrm{U}$ NDERSTADING the neurophysiological signals underlying voluntary motor control and decoding them for controlling limb prostheses are among the major challenges in applied neuroscience and rehabilitation engineering. Individuals with a below-elbow amputation

This work was partially supported by the European Commission under the WAY project (FP7-ICT-288551), by INAIL (the Italian national workers' compensation) under the PPR3 project and the Swedish Research Council (VR 2016-01635). The work of C. Cipriani was partially supported by the European Research Council under the MYKI project (ERC-2015-StG, Grant no. 679820).

G. Kanitz, and C. Cipriani, are with The BioRobotics Institute, Scuola Superiore Sant'Anna, V.le Piaggio 34, 56025 Pontedera (PI) Italy (e-mail: ch.cipriani@santannapisa.it). B. B. Edin is with the Department of Integrative Medical Biology, Physiology Section, Umeå University, SE-901 87 Umeå, Sweden. maintain part of the 18 extrinsic muscles that originally served the fingers and wrist. The electromyogram (EMG) recorded from these muscles can in theory be used to control a variety of grasps and movements in a multi-digit hand prosthesis. Although other promising techniques have been proposed and assessed, like peripheral electrodes [1], [2], implantable epimysial electrodes [3] or myoelectric sensors [4] and targeted muscle reinnervation [5], the use of EMG signals recorded from the skin surface remains the most widely spread, reliable and clinically viable approach for controlling hand prostheses [6]. Remarkably, the most reliable controller available today is substantially the two-state amplitude modulation EMG controller proposed by Bottomley [7] back in the ' $60 \mathrm{~s}$ in which a single pair of agonist/antagonist muscles controls the opening and closing of the prosthetic hand. However, this scheme cannot differentiate between different muscular patterns pertaining to different hand movements, and, accordingly, cannot be used to control multiple grasps of a dexterous prosthesis.

An alternative approach is pattern recognition, as first proposed by Finley and Wirta in 1967 [8]. This technique is based on the premise that amputees can activate repeatable and distinct muscular contractions for each class of desired motion and that the associated EMG patterns can be used to send commands to the prosthesis. Ideally, this occurs in a physiologically appropriate manner, i.e., that the individual contracts the residual muscles as to perform a particular grasp that the prosthesis then implements. Englehart and colleagues pioneered the development of continuous classifiers [9]-[11] that still represent the state of the art. With these classifiers, a set of statistical features is extracted from a continuous stream of signals using a sliding window at a certain rate [12]. The features are then fed into a classification algorithm able to differentiate between different patterns of muscle contractions and to produce a new decision (output of the classifier) at every time step, in a continuous fashion, with latencies short enough to be perceived as real time by the individual (typically within $300 \mathrm{~ms}$ [13]). Researchers have demonstrated that these classifiers are able to discriminate up to 12 hand/wrist movements from forearm EMGs, with accuracies $>95 \%$ both offline and in constrained laboratory environments [14]-[17]. However, if such classifiers also can be successfully applied in realistic settings, i.e., with different arm postures, grasps and force levels, remains to be resolved [18]-[20].

Remarkably, the assumption that repeated muscular contractions produce repeatable patterns of steady-state EMGs is weak. In fact, the steady-state EMG has very little temporal structure (it is mostly a random signal) due to the active 
modification of recruitment and firing patterns needed to sustain the contraction [21], [22]. In addition to this neurophysiological factor, changes in skin-electrode impedance, electrode shifts and varying loads on the residual limb [6] further affect the temporal structure of the EMG signal. For these reasons, time-averaged, compound statistical properties have to be extracted from the EMG signals for the classification. To further improve the reliability of the classification, majority voting (MV) is usually applied to the output of the continuous classifiers, such that the output class at a given time step is the most common class in the previous $n$ time steps [10]. More complex post-processing approaches, such as velocity ramp (VR) [23] or confident-based rejection (CBR) methods [24] also exist. In short, continuous classifiers require both dimension reduction methods (statistical features extraction) and time domain filters (MV, VR or CBR behave as low pass filters), yet they are intrinsically prone to errors. Not surprisingly, the only clinically available continuous classifier based on pattern recognition is trained to recognize just two classes/movements [25].

While investigating the properties of the EMG coincident with the onset of rapid contractions, Hudgins and colleagues observed a substantial degree of structure in the transient waveforms of upper arm muscles [26]. This observable structure has been reported by others [27], and suggests an orderly recruitment of motor units [22]. Other investigators assessed the performance of different classifiers, seeking to improve the accuracy of Hudgins' and colleagues' method [28]-[30], and very recently Jiang et. al. proposed a classification strategy following the same principles [31]. Besides these few studies, to our knowledge this approach was not further investigated and this work aimed to bridge this gap.

Building on Hudgins seminal work, here we propose an approach for decoding the intended grasp from the forearm EMGs by processing the signals associated with the onset of muscle contraction. As grasping largely relies on feedforward mechanisms [32] we hypothesized that the muscle contractions associated with the initial phase of the grasp contained predictive information about the intended, preplanned grasp. If this hypothesis is correct it should be possible to decode the intended grasp only once during a reach-for-grasp action, thus avoiding the instability effects associated with continuous classification, and well in advance compared to the latter. To assess our hypothesis, we collected EMG data from the forearms of able-bodied participants and hand amputees while they performed four hand movements. The movements chosen, i.e., power, pinch, and lateral grasps, and hand opening include grasps that cover $\sim 80 \%$ of activities of daily living [33] and can be implemented by clinically available multi-grasp hands [34]. The movements were performed in nine different positions within the work-space of the arm. Through off-line processing we extracted the portions (windows) of data associated to the onset of the muscular contraction from the EMG recordings, i.e., the transition between no-contraction and contraction. We processed such portions of data (or transients) to identify the intended movements using a representative classifier. Besides assessing the viability of the approach, we searched for the input parameters (window length and sampling frequency) that optimized the performance independently of the arm position.

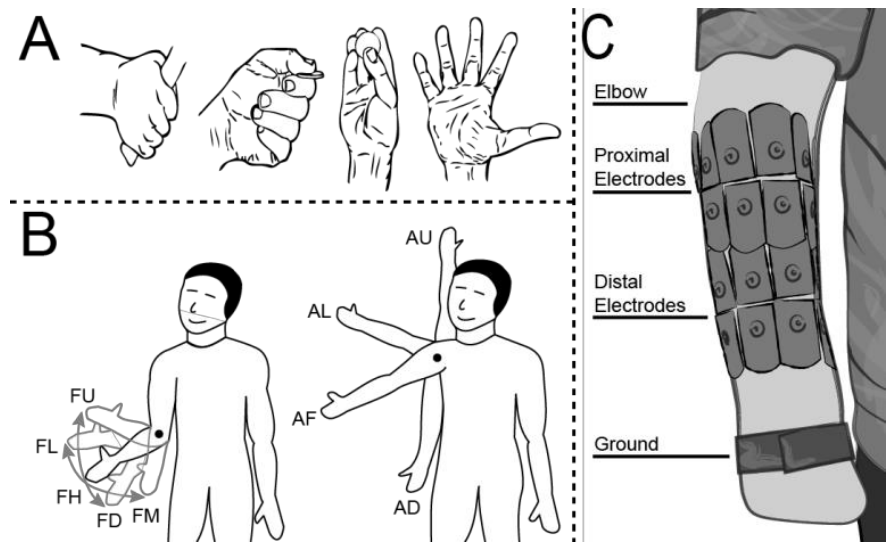

Fig. 1 Experimental setup. A) Four hand movements: power grasp, lateral grasp, pinch grasp, and hand open. B) Nine arm positions: elbow flexed in the parasagittal plane at $\sim 45^{\circ}$ (FD), $\sim 90^{\circ}$ (FH) and $\sim 135^{\circ}$ (FU); elbow flexed at $\sim 90^{\circ}$ with the shoulder medially rotated $\sim 45^{\circ}$ (FM), and laterally rotated $\sim 45^{\circ}$ (FL) (left picture); arm reaching down $(A D)$, reaching up $(A U)$, reaching the front $(A F)$, reaching lateral (AL) (right picture). C) Placement of the 15 electrodes around the circumference of the forearm.

The configuration that proved optimal off-line was implemented in a real-time system operating with clinically widely available EMG sensors and successfully demonstrated by one amputee operating a multi-grasp prosthetic hand.

\section{MATERIALS AND METHODS}

\section{A. Participants and experimental protocol}

Ten participants took part in the experiments after giving their informed consent. Two participants (both males) were below-elbow amputees and myoelectric hand users (Table I). The eight able-bodied volunteers (aged 24-46, two females) had no history of neuromuscular disorders.

Participants were instructed to stand upright and to perform four different hand movements: a power grasp, a lateral thumb-index grasp, a three-digit pinch grasp, and hand opening (Fig. 1A). These movements were performed at nine different arm positions including four with the elbow fully extended and five with the elbow flexed (arm reaching the front, lateral, up and down). Fully extended elbow positions included: arm reaching down, up, front, and lateral. In three of the elbow flexed positions the elbow was flexed in a parasagittal plane (crossing the shoulder) at $\sim 45^{\circ}, \sim 90^{\circ}$, and $\sim 135^{\circ}$; in the last two the elbow was flexed at $\sim 90^{\circ}$ with the shoulder/humerus medially rotated $\sim 45^{\circ}$ ) or with the shoulder/humerus laterally rotated $\sim 45^{\circ}$ (Fig. 1B). This variability was included to assess the robustness of the decoding approach with respect to arm positions and covered a large portion of the normal work space. Each of the four hand movements was repeated five times in a randomized order in each of the nine arm positions, for a total of 180 repetitions. Instructions on what arm position to adopt and which movement to perform were visually presented on a screen along with a progress bar that indicated the duration (3 seconds) and remaining time. The participants were instructed to perform the grasps at moderate, non-fatiguing force levels and were allowed to rest after each trial. 
Table I Demographic Data of Below-Elbow Amputees

\begin{tabular}{|c|c|c|c|c|c|c|c|}
\hline ID & Age & $\begin{array}{l}\text { Years since } \\
\text { Amputation }\end{array}$ & Cause & Side & Level & $\begin{array}{c}\text { Residual limb, } \\
\text { length from elbow }\end{array}$ & $\begin{array}{c}\text { Contralateral forearm } \\
\text { (from elbow) }\end{array}$ \\
\hline A1 & 38 & 21 & traumatic & Right & Wrist & $35 \mathrm{~cm}$ & $51 \mathrm{~cm}$ \\
\hline A2 & 44 & 20 & traumatic & Right & Transradial & $22 \mathrm{~cm}$ & $42 \mathrm{~cm}$ \\
\hline
\end{tabular}

Fifteen differential channels of surface EMGs were recorded using disposable $\mathrm{Ag} / \mathrm{AgCl}$ electrodes with an interelectrode distance of $2 \mathrm{~cm}$ at a sampling rate of $2048 \mathrm{~Hz}$ (using the EMG-USB2+, OT BioElettronica S.r.l., Turin, Italy) and stored for offline analysis. For both able-bodied and amputees, the surface electrodes were placed equidistant in a cuff fashion around the forearm starting just distal to the elbow joint (Fig. 1C) to cover most extrinsic flexor and extensor muscles of the hand (i.e., extensor digitorum communis, flexor digitorum superficialis, abductor pollicis longus, extensor pollicis longus, and flexor pollicis longus). The general purpose of the placement was to obtain recordings from as many different muscles as possible.

Able-bodied participants were fitted with an 18 joint sensors data-glove (CyberGlove III by Cyber Glove Systems LLC, San Jose, CA; acquisition rate $30 \mathrm{~Hz}$ ). The glove was used as a reference to verify the possibility of detecting the onset of contraction from the EMGs with respect to the actual hand and finger movements. EMG data and hand movements were synchronized using a data acquisition board (USB-6009, National Instruments Corporation, Austin, TX) and a custom application running on a laptop. The recorded data was analyzed offline.

\section{B. Data analysis}

The goal of the data analysis was twofold: (i) to assess whether different hand movements could be classified using a window of EMG data containing the onset of the contraction; and (ii), to identify time-domain parameters (such as the length of the window and the sampling frequency) that optimized the performance of such a classification scheme.

The data analysis proceeded in several stages (Fig. 2). First, the entire raw EMG signal was whitened using parameters from the training set [35] and then the mean absolute value (MAV) was extracted from each channel of raw EMG signal, using an overlapping sliding window of $100 \mathrm{~ms}$ with a single sample displacement $(1 / 2048 \mathrm{~Hz})$ [12]. Second, the MAV was filtered and down-sampled to one out of five sampling frequencies $\left(\mathrm{F}_{\mathrm{S}}\right)$ under investigation: 100, 80, 60, 40 and 20 $\mathrm{Hz}$. During reach-for-grasping actions in daily-life actions, the hand is preshaped long before the hand is close to the target object [36]. The associated onset of forearm contractions, $t_{T}$, was identified for each piece of data associated to each movement by means of an onset detection algorithm (ODA) described below. Finally, the processed EMG signals in a time window (transient window) starting at $\mathrm{t}_{\mathrm{T}}$ and lasting for $\mathrm{W}_{\mathrm{L}}$ (window length), were fed into a classifier. $\mathrm{W}_{\mathrm{L}}$ was varied from 0 to $300 \mathrm{~ms}$ in steps of $50 \mathrm{~ms}$.

The ratio of windows properly associated to the target movement, i.e., the true positive rate (TPR; Equation (E1)), was used as the metric to assess the performance of the classification scheme and to infer its sensitivity with respect to the amount of the input data $\left(\mathrm{W}_{\mathrm{L}}\right)$ and its sampling frequency $\left(\mathrm{F}_{\mathrm{S}}\right)$.

$$
T P R=\frac{T P}{\text { Trials }}
$$

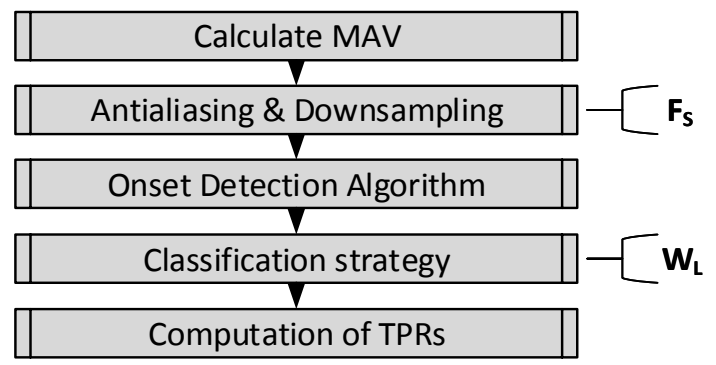

Fig. 2 Steps of the data analysis. Acronyms: MAV - Mean Absolute Value of EMG; TPR: True Positive Rate. $F_{S}$ : Sampling frequency. $W_{L}$ : Window length.

1) Choice of Choice of window length and sampling frequency

The choice of $\mathrm{W}_{\mathrm{L}}$ and $\mathrm{F}_{\mathrm{S}}$ can be crucial because they influence the response time and possibly the accuracy of the classification [37]. For each participant and each combination of $\mathrm{W}_{\mathrm{L}}$ and $\mathrm{F}_{\mathrm{S}}$, EMGs collected in a single arm position were used to train the classifier.

The data from the remaining eight arm positions were used for the evaluation of the TPR (leave-8-out validation). This procedure was repeated for all 9 arm positions to compute a global $\mathrm{W}_{\mathrm{L}}$ and $\mathrm{F}_{\mathrm{S}}$ dependent TPR for each participant.

The combination of $\mathrm{W}_{\mathrm{L}}$ and $\mathrm{F}_{\mathrm{S}}$ that yielded to optimal TPR, was found by fitting the TPR across all participants with a multivariate $2^{\text {nd }}$ degree polynomial regression model with interaction terms (E2). Regression coefficients $\left(\mathrm{c}_{1}, \ldots, \mathrm{c}_{6}\right)$ which did not show statistical significance $(\mathrm{p}>0.05)$ were ignored.

$$
\begin{gathered}
T P R_{R E G}=c_{1}+c_{2} W_{L}+c_{3} F_{S}+c_{4} W_{L} F_{S} \\
+c_{5} W_{L}^{2}+c_{6} F_{S}^{2}
\end{gathered}
$$

The maximum acceptable classification delay with respect to the onset (coincident with the window length, $\mathrm{W}_{\mathrm{L}}$ ), was limited to $300 \mathrm{~ms}$ to comply with real-time constraints [13]. A Friedman test was finally used to infer on statistical differences between the optimal combination and all the others.

The combination of parameters $\left(\mathrm{W}_{\mathrm{L}}{ }^{*}, \mathrm{~F}_{\mathrm{S}}{ }^{*}\right)$ that proved optimal across all participants with respect to performance (TPR) and maximum classification delay ( $\left.\mathrm{W}_{\mathrm{L}} \mathrm{MAX}\right)$, was finally used to infer on the robustness of the approach across 
arm positions. To this end, we computed for each participant the performance of the trained classifier in all each arm position and tested in the remaining ones. Based on these results, we determined the optimal training position and finally computed the TPR across arm positions.

\section{2) Onset Detection Algorithm (ODA)}

The onset of the muscle contraction $\left(\mathrm{t}_{\mathrm{T}}\right)$ was retrieved from the EMG data associated with each movement by means of a threshold detector. The threshold was computed using the training set and was determined using a test signal $\left(\mathrm{MAV}_{\mathrm{TST}}\right)$, computed as the sum of the EMG channels (MAV), with each channel previously high-pass filtered for offset removal, as in:

$$
\operatorname{MAV}_{\mathrm{TST}}(t)=\sum_{c h=1}^{n_{E M G}}\left(M A V(t, c h)-\frac{\sum_{t_{O}=t-0.3 s}^{t} M A V\left(t_{O}, c h\right)}{0.3 \cdot F s}\right)
$$

The duration of the filter window $(300 \mathrm{~ms})$ was chosen to reduce the distortion of the onset as in Hudgins and colleagues [26]. $t_{\mathrm{T}}$ was fixed at $50 \%$ the minimum peak amplitude reached by $M A V_{\text {TST }}$ across all trials in the training set. We chose this rather simple ODA instead of more complex, state of the art ODAs (e.g. the Teager-Kaiser energy operator [38]) as it could be implemented online with limited computational cost.

The onsets of the physical movements in able-bodied subjects $\left(\mathrm{t}_{\mathrm{G}}\right)$ were also identified in a similar manner using the glove data. Another test signal, $\mathrm{GLV}_{\mathrm{TST}}$, was computed (E4) and the threshold was set at $50 \%$ the peak velocity.

$$
\operatorname{GLV}_{\mathrm{TST}}(t)=\sum_{c h=1}^{n_{G L V}} a b s\left(\frac{d}{d t} G L V(t, c h)\right)
$$

The median and the central $50^{\text {th }}$ inter-percentile range of the difference between the onsets detected from the EMG $\left(t_{T}\right)$ and the data glove $\left(t_{\mathrm{G}}\right)$ were used, as an indirect yet qualitative measure, to infer on the accuracy and precision of the EMG ODA.

This ODA was found to be working well but there are certainly other useful alternatives [38]-[41].

\section{3) Classification scheme}

We have no reason to believe that there is a specific classifier that outperforms all others. However, we implemented a state of the art Error-Correcting Output-Codes classifier [42] with a one-versus-all coding matrix [43], comprising four binary support vector machines using a linear kernel. Unlike conventional continuous classification schemes, our classifier was fed with time series of EMG windows starting at $t_{T}$ and lasting for $\mathrm{W}_{\mathrm{L}}$ seconds. The classifier was fed with vectors $\left(\mathrm{V}_{\mathrm{T}}\right)$ that contained the temporal evolution of the signals, rather than only instantaneous patterns as in conventional schemes (Fig. 3) [44].

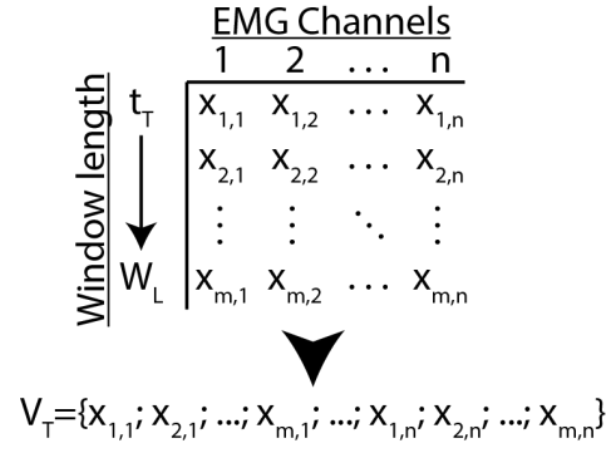

Fig. 3 Representation of the input data used for classification. The extracted EMG window (nxm matrix) is transformed into a row vector $\left(\mathbf{V}_{\mathrm{T}} \in \mathrm{R}^{1 \times \mathrm{n} \cdot \mathrm{m}}\right)$ and used for classification.

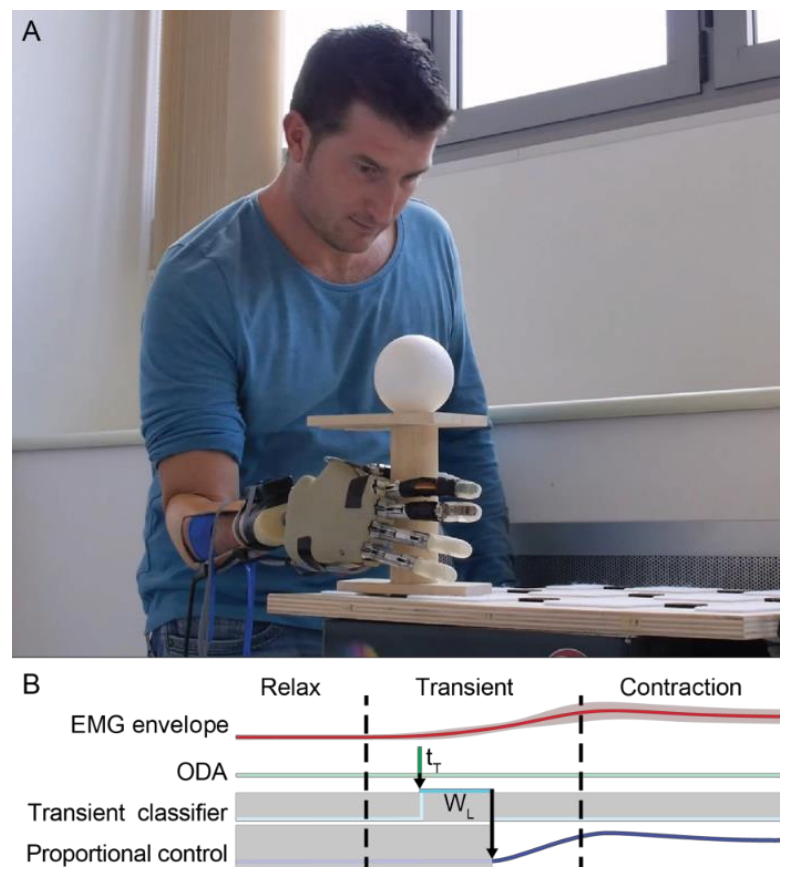

Fig. 4 Experimental setup of the online system. A) Individual with below elbow amputation wearing the research prosthesis. B) Online implementation of the transient EMG classifier. Once the transient detection algorithm (ODA) identifies an onset (at $\mathrm{t}_{\mathrm{T}}$ ), the transient window $\left(\mathrm{W}_{\mathrm{L}}\right)$ is recorded and classified; thereafter, the average of the (steady-state) EMG signals from four EMG sensors is computed and used to proportionally modulate the aperture of the identified grasp.

\section{Online implementation and feasibility test}

The proposed approach was implemented in an actual research prosthesis and its performance in real-time was qualitatively assessed in a realistic workspace and condition. The prosthesis included a customized prosthetic socket with four EMG sensors (Otto Bock, 13E125=50, Vienna, Austria), a research robotic hand (IH2 Azzurra by Prensilia S.r.l., Pontedera, Italy), and a portable micro PC (Sharks Cove by Microsoft, Redmond, WA; Intel ATOM Processor, 4 Core, $1.88 \mathrm{GHz}$ and $1 \mathrm{~GB}$ of RAM) running a Matlab application that implemented the classification scheme (Fig. 4A). The socket was manufactured for one hand amputee (participant A1 - Table I). The participant was an experienced, regular user of a conventional myoelectric hand but had limited experience of multi-grasp hands. A proportional velocity 
control, having the mean EMG signal as the input, was used to control the hand aperture once a hand movement (one of the four) had been classified (Fig. 4B). The participant was asked to use the three grips to transport three objects from one position to another on a stand or on a table. The task required large arm movements. Prior to the actual test, the participant performed a short session ( $\sim 30$ seconds) during which both the classifier and the participant got trained (supplementary video). The threshold used for the ODA was computed using the data recorded during this training session.

\section{Results}

For each participant the experimental recordings lasted for less than an hour including the setup preparation. The implemented ODA proved functional in identifying the onset of the contraction with respect to the actual physical movement as recorded by the data-glove in able-bodied. In particular the $t_{T}$ was mostly detected before $t_{G}$ albeit varied largely across participants and hand movements (Fig. 5). The onset detected from the EMG $\left(\mathrm{t}_{\mathrm{T}}\right)$ anticipated the physical movement $\left(\mathrm{t}_{\mathrm{G}}\right)$ in the median by $120,190,250$, and $70 \mathrm{~ms}$, for the pinch, open, power and lateral movements, respectively.

The fitting of the TPR with a $2^{\text {nd }}$ order polynomial (E2), proved $F_{S}$ and its interaction with $\mathrm{W}_{\mathrm{L}}$ to be not statistically significant $(\mathrm{p}>0.05)$. In other words different sampling frequencies did not affect the TPR of the classifier $\left(\mathrm{F}_{\mathrm{S}}{ }^{*} \in\right.$ $[100,80,60,40,20] \mathrm{Hz})$. Accordingly, we used a model of the TPR (E2) that only depended on $\mathrm{W}_{\mathrm{L}}$ :

$$
T P R_{R E G^{*}}=c_{1}+c_{2} W_{L}+c_{5} W_{L}^{2}
$$

For the remaining analysis, the results of the classification across all sampling rates were combined to increase the sample size.

The TPR fitting across all participants proved optimal (with a TPR of $94 \%$ ) for $\mathrm{W}_{\mathrm{L}}=\mathrm{W}_{\mathrm{L}} *=300 \mathrm{~ms}$ (Fig. 6). However, the Friedman test revealed no statistical differences between the result at $\mathrm{W}_{\mathrm{L}}{ }^{*}$ and those for $\mathrm{W}_{\mathrm{L}} \geq 150 \mathrm{~ms}$.

The TPRs of the classifier when trained in each arm position and tested in the remaining ones, was recomputed for each participant, using the optimal configuration (i.e., $\mathrm{W}_{\mathrm{L}}{ }^{*}=$ $300 \mathrm{~ms}$ ). Across all participants, the FD training position (elbow $\sim 45^{\circ}$ flexed in the parasagittal plane) resulted in the best classification of the four hand movements; all training positions also resulted in an overall TPR $\geq 89 \%$ (Fig. 7A). With FD as the training position, the performance of the classifier remained largely invariant to changes in arm position (Fig. 7B). When training in the FD position and testing in the remaining arm positions the lateral grasp was prone to errors in the AU (elbow extended, arm reaching lateral) positions, whereas the pinch grasp had a reduced performance for the AF (elbow extended, arm reaching front) position (Fig. 7B). Finally, using $\mathrm{W}_{\mathrm{L}}{ }^{*}, \mathrm{~F}_{\mathrm{S}}{ }^{*}$, training in the FD position and testing in all other arm positions, yielded an average TPRs of $96 \%$ for able-bodied participants and $95 \%$ for amputees (Fig. 8; for the two amputees the average TPR was $93 \%$ and $97 \%$, respectively ).

The outcomes from the online implementation and feasibility test are preliminary and qualitative in nature. However, the online myocontroller decoding grasp-type and grip force (proportional control) appeared feasible and robust in a realistic scenario (supplementary video). Following a short training of the classifier, the participant was able to operate the hand and successfully transfer objects using the three implemented grasps, in a wide range of the arm workspace (Fig. 9; supplementary video). The classifier trained in one position thus proved robust in all other positions. Importantly, the control strategy we propose evidently does not critically depend on a large number of EMG electrodes - at least with a limited set of hand movements-since the amputee successfully managed to control the prosthetic hand with four rather than 15 electrodes.

\section{Discussion}

To summarize, we claim that forearm EMGs patterns at the onset of a contraction contain predictive information about upcoming grasps and, as such, can be used for real-time control of a prosthetic hand.

The idea of using pattern recognition of transient EMGs for prosthetic control is not new. Hudgins and colleagues from University of New Brunswick (UNB) [26] proposed already in 1993 the use of transient EMG signals at the onset of a muscle contraction (of upper arm muscles). However, in their following studies in 2001 [9] and 2003 [10], they abandoned the transient approach in favor of the continuous classification strategy claiming that the requirement of "initiating $a$ contraction from rest ... prohibits switching from class to class in an effective or intuitive manner" [10]. Their continuous classifier had a major impact in the field of upper limb prosthetics research and has been the starting point for the vast majority of pattern recognition control approaches/systems developed worldwide since then ${ }^{1}$. Still, due to the nature of the steady-state EMG [21], [22] continuous classifiers are known to suffer from instability issues, which require post-processing techniques to address them [10], [23], [45]. Post-processing algorithms inevitably reduce the responsiveness of the system as they infer how to remove misclassified samples at the output of the classifier from history or prior information. The transient EMG approach uses only the data contained in a short window associated to a muscle contraction, which is known to contain a deterministic structure [26], [27]. The advantage of this approach is that classification is only necessary when a transient window is detected by the ODA, making the entire system less prone to errors. In addition, when errors occur, it is comparatively simple for the user to abort the ongoing grasp attempt and start anew. Importantly, since the contraction precedes the actual movement (Fig. 5), the response time of the transient classifier is faster than that of a conventional continuous classifier.

\footnotetext{
${ }^{1}$ It would be impractical to cite all the systems developed based on [9] and [10]. At the time of writing this manuscript [9] has been cited $>500$ times, and [10] $>900$ times.
} 


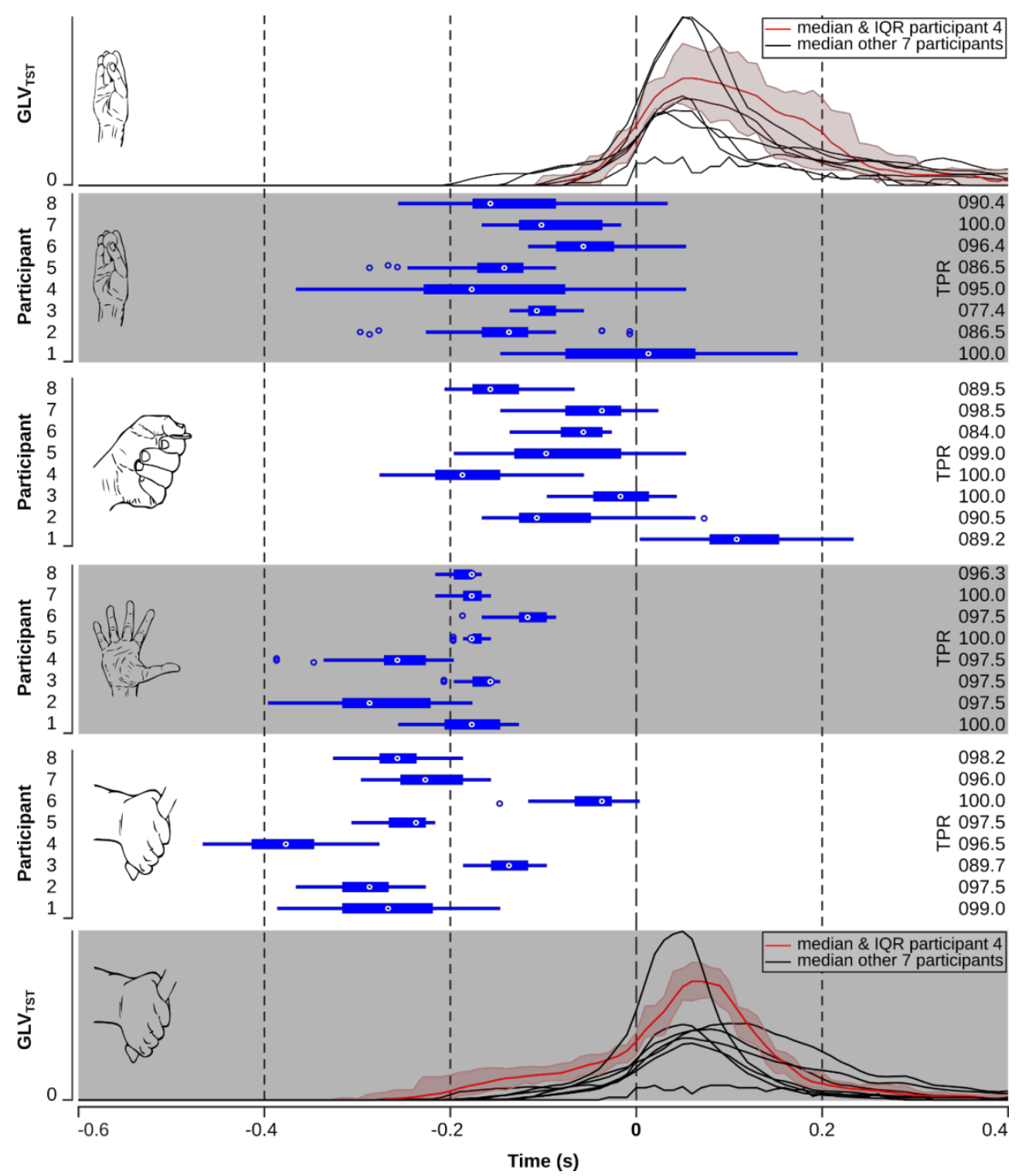

Fig. 5 Accuracy and precision of the ODA. The top and bottom panels show the median velocity profiles from the data glove (GLV $\mathrm{TST}_{\text {TS }}$ across all able-bodied participants for the pinch (top panel) and the power grasp (bottom panel). Intra-subject variability is illustrated by the interquartile range (IQR) for a single participant, i.e., participant 4 (in red). The middle four panels show for each type of grasp onsets as detected by the EMG $\left(t_{T}\right)$ relative to the glove $\left(t_{G}=0 \mathrm{~s}\right)$. On each box, the central mark is the median, the edges of the box are the 25th and 75 th percentiles, the whiskers extend to the most extreme data points not considering outliers, and outliers are plotted individually. The average true positive rate (TPR) for each participant is found to the right.

The choice of the UNB group to abandon the transient approach in favor of the continuous one was likely influenced by the prosthetic components available at the time, and in turn by the limb movements under investigation. These were wrist movements (flexion/extension and radial/ulnar deviation) plus opening/closing of the hand, all digits together [9]. The transient approach required an individual to elicit a contraction from rest but this was a rather awkward imposition when performing sequences of such movements. Hence it was deemed appropriate to abandon the transient approach although this entailed using the less deterministic steady-state EMG signals. Today multi-Degrees of Freedom (DoF) hands are able to perform different grasp types, not just the opening and closing of all the digits together exist [34]. If the aim is to decode such grasps from the EMG signals - and this was our goal - it is perfectly acceptable to conceive a control scheme in which the different grasps can be formed starting from a relax state of the prosthesis but not from any other state/grasp [34].
During normal reach-to-grasp movements humans start from a 'rest' state and then as they reach to grasp an object, they gradually mould the hand to enclose it using a predetermined grasp type (e.g. lateral grasp, pinch grip, etc.) and stereotypical movements [46], [47]. For simple grasping tasks, the grasp type is maintained until the hand is reopened. Moreover, available multi-DoF hands do not even allow complex in-hand manipulation. It thus seems reasonable to propose a control scheme in which different grasps are formed starting from a relax state of the prosthesis but not from any other state/grasp. The transient approach proposed by Hudgins and colleagues finds in the grasp control of prosthetic hands for below-elbow amputees a new and apt application. Interestingly, this approach was recently investigated by Jiang et. al. [31] who proposed a transient classification strategy (they used the term transition) for the control of a prosthesis which included a single DoF hand and a wrist rotator, achieving results in line and complementary with our work. 


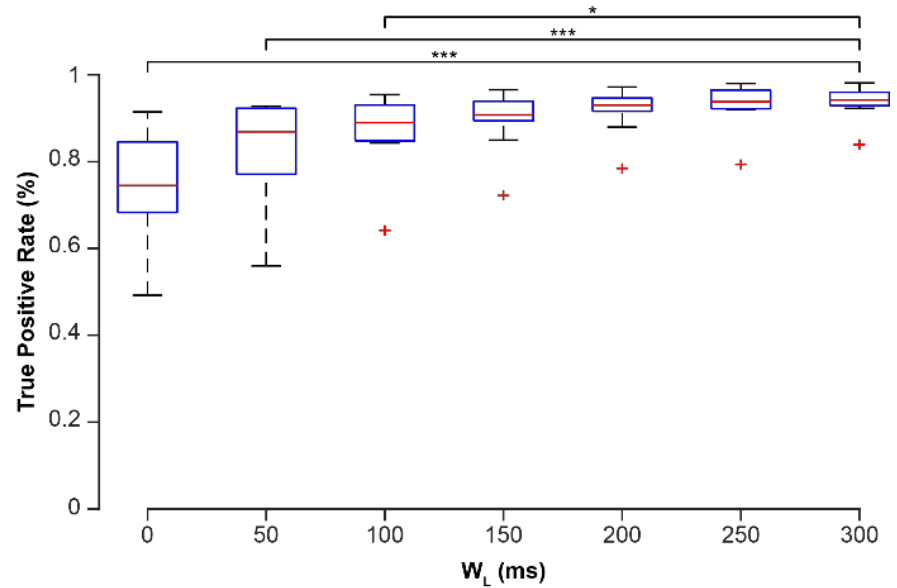

Fig. 6 Boxplot of the true positive rates (TPRs) as function of WL with indications of groups that show a statistically significant difference from the optimal $\mathrm{WL}\left(\mathrm{WL}^{*}=300 \mathrm{~ms}\right)$ as determined by the Friedman test ${ }^{*}$ : $0.05 \geq p>0.01 ;{ }^{* *}: 0.01 \geq p>0.001 ;{ }^{* * *}: 0.001 \geq p$ ).

The performance of the transient classifier relies on the ability of the ODA to identify the onset of a muscle contraction, and thus to feed the classifier with the portion of data which contains the temporal pattern associated to a certain movement. Our recordings with able-bodied participants, showed that our simple EMG ODA detected onsets ahead of the movements but with some variability across movements and participants. However this variability was mainly inter-participant and the individually-trained ODA was able to capture the intended grasp (Fig. 5). Nonetheless, the ODA used in this study should be considered just an example; ODAs with better temporal precision may further improve the accuracy of the classifier.

The optimization of the parameters $\mathrm{W}_{\mathrm{L}}$ and $\mathrm{F}_{\mathrm{S}}$ yielded to interesting results. Specifically, the performance was not statistically different with window lengths $\geq 150 \mathrm{~ms}$ (Fig. 6). This is highly relevant for clinically viable control system for prostheses. It suggests that the EMG signals picked up by conventional myoelectric sensors (e.g. Otto Bock, $13 \mathrm{E} 125=50$ ), which are a rectified and low-pass filtered version of the raw signal (similar to the MAV used in this work) could be sampled at a relatively low sampling frequency, with reduced computational cost. It also suggests that a window of data containing $\geq 150 \mathrm{~ms}$ (i.e., down to $\sim 4$ samples, at $F_{\mathrm{S}}=20 \mathrm{~Hz}$ ) is enough for successful classification; as this window starts before the actual movement the response of the classifier might well be perceived as in real-time.

The analysis of the TPR across arm positions proved the transient EMG approach resistant to the limb position effect [18], [20], currently observed in steady-state classifiers. In practice, changes of arm posture and of applied loads to the prosthesis, generate relative movements between the stump and the socket, that yield to changes in the recorded EMG, which in turn is misclassified [18], [48], [49]. Current approaches for reducing such an effect include the use of orientation sensors and/or increasing the sample of training set from different limb positions [48] or the application of computationally expensive features [50]. Our analysis, confirmed by the online test, suggests that a transient EMG classifier requires no additional hardware (or computation, or training time) to achieve consistent and successful operation in the arm workspace. Again, this is important in clinical prosthetics).

It is known that multiple time domain features improve the accuracy of the classification, and that optimal feature sets do exist for continuous classifiers [10], [37], [44]. Nonetheless we decided to use a single feature here, i.e., MAV. This choice was primarily dictated by the fact that the MAV of the raw EMG is known to be comparable to the output of clinically available EMG sensors. This allowed us to infer on the applicability of our outcomes to a more applied scenario and to test the algorithms in a real-time experimental setup. The latter, although not providing quantitative results (and this represents a limitation for the present study), confirmed the results of the offline analysis proving that the transient classifier could work in a clinical setup. It should be noted that in the case of real-time control (i.e., with a user in the loop), the user would likely adapt to the system and learn how to achieve optimal performance [51]. Nonetheless it needs to be clarified how more complex feature sets [10], [26], [52] or classifiers, would perform with the present approach, offline and in real-time. The generalizability of the approach also needs to be investigated, as classification performance generally degrades with the number of classes/movements.
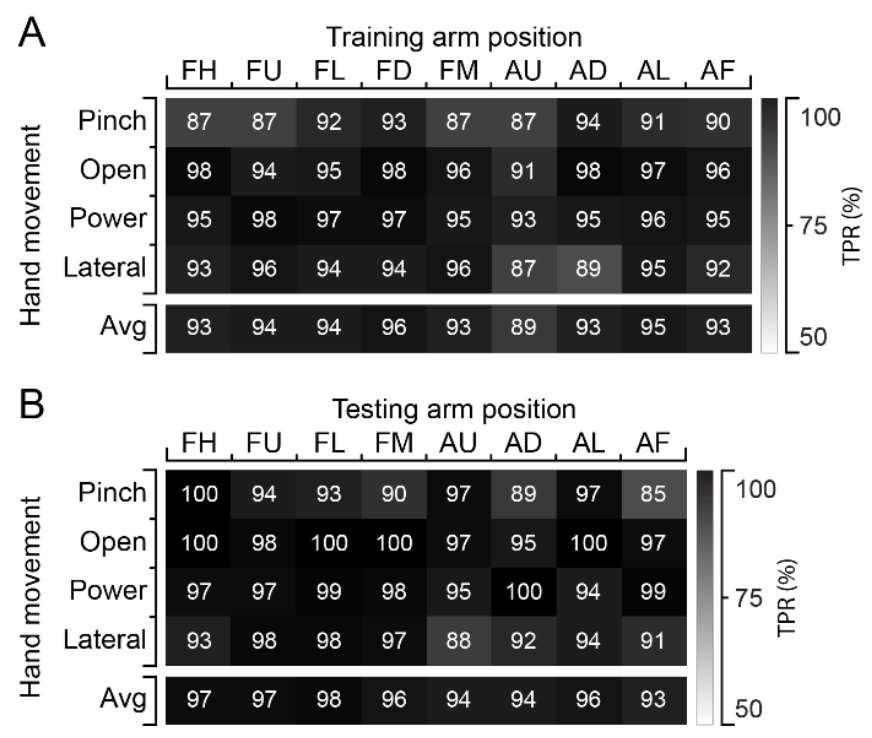

Fig. 7 True Positive Rates (TPRs) for each hand movement using the found optimal parameters $\left(\mathrm{WL}^{*}=300 \mathrm{~ms}, \mathrm{FS}^{*}=\in[100,80,60,40\right.$, 20] $\mathrm{Hz}$ ) (results from all participants). A) TPRs based on training positions. B) TPRs using the optimal training position (FD). The bottom rows depict the average TPRs across movements. Position acronyms: elbow flexed in the parasagittal plane at $\sim 45^{\circ}$ (FD), $\sim 90^{\circ}$ (FH) and $\sim 135^{\circ}$ (FU); elbow flexed at $\sim 90^{\circ}$ with the shoulder medially rotated $\sim 45^{\circ}$ (FM), and laterally rotated $\sim 45^{\circ}(\mathrm{FL})$; arm reaching down (AD), reaching up $(A U)$, reaching the front $(A F)$, and reaching lateral $(A L)$. 


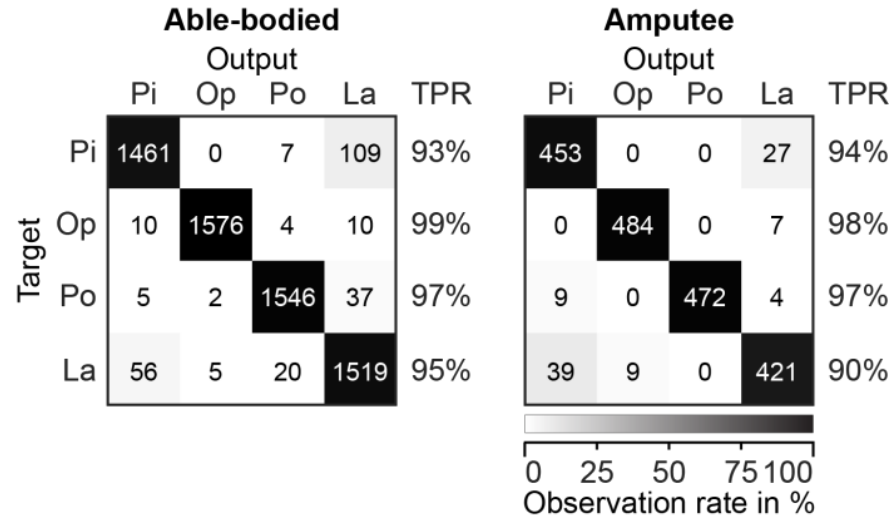

Fig. 8 Hand movements confusion matrices using optimal parameters (training in down position, $\mathrm{WL}^{*}=300 \mathrm{~ms}, \mathrm{FS}^{*}=\in[100,80,60,40,20]$ $\mathrm{Hz}$ ). The TP is in the diagonal. The TPR for each target class is indicated on the right of each matrix.

Finally, the results achieved in this work invite studies of a number of questions related to the transient EMG approach. It is not known if it can be applied to control simultaneously hand and wrist motions, nor if it can be used to estimate the grip force for the desired grasp. To assess the clinical viability of the transient approach a study with multiple participants and varying levels of below-elbow amputation, operating actual prostheses, and comparing the transient approach with a state of the art continuous classifier would be desirable.

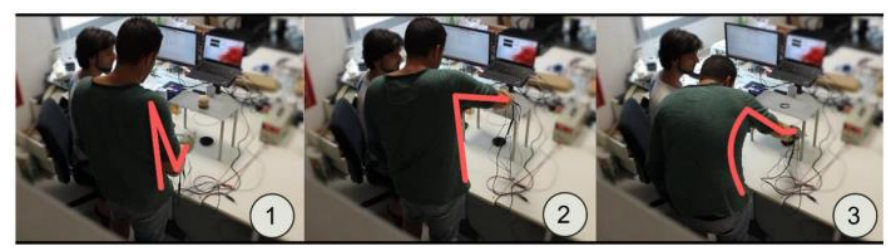

Fig. 9 Snapshots from the feasibility tests of the online myoelectric controller. This required the participant to perform large movements of the upper limb to transfer objects that required various grasps (cf., the video clip in the supplementary materials).

\section{REFERENCES}

[1] P. M. Rossini et al., "Double nerve intraneural interface implant on a human amputee for robotic hand control," Clin. Neurophysiol., vol. 121, no. 5, pp. 777-783, 2010.

[2] G. S. Dhillon and K. W. Horch, "Direct Neural Sensory Feedback and Control of a Prosthetic Arm," IEEE Trans. Neural Syst. Rehabil. Eng., vol. 13, no. 4, pp. 468-472, 2005.

[3] M. J. Ortiz-Catalan, B. Håkansson, and R. Brånemark, "An osseointegrated human-machine gateway for long-term sensory feedback and motor control of artificial limbs," Sci. Transl. Med., vol. 6, no. 257 , p. 257 re6-257re6, 2014

[4] P. F. Pasquina et al., "First-in-man demonstration of a fully implanted myoelectric sensors system to control an advanced electromechanical prosthetic hand," J. Neurosci. Methods, vol. 244, pp. 85-93, 2015.

[5] T. A. Kuiken et al., "Targeted Muscle Reinnervation for Real-time Myoelectric Control of Multifunction Artificial Arms," J. Am. Med. Assoc., vol. 301, no. 6, pp. 619-628, 2009.

[6] N. Jiang, S. Dosen, K. R. Muller, and D. Farina, "Myoelectric Control of Artificial Limbs: Is There a Need to Change Focus?," IEEE Signal Process. Mag., vol. 29, no. 5, pp. 150-152, 2012.

[7] A. H. Bottomley, "Myoelectric Control of Powered Prostheses," Bone Joint J., vol. 47, no. 3, pp. 411-415, 1965.
[8] F. R. Finley and R. W. Wirta, "Myocoder studies of multiple myopotential response," Arch. Phys. Med. Rehabil., vol. 48, no. 11, p. 598, 1967.

[9] K. Englehart, B. Hudgins, and P. A. Parker, "A wavelet-based continuous classification scheme for multifunction myoelectric control," IEEE Trans. Biomed. Eng., vol. 48, no. 3, pp. 302-311, 2001.

[10] K. B. Englehart and B. Hudgins, "A robust, real-time control scheme for multifunction myoelectric control," IEEE Trans. Biomed. Eng., vol. 50, no. 7, pp. 848-54, 2003.

[11] G. Li, A. E. Schultz, and T. A. Kuiken, "Quantifying pattern recognition-based myoelectric control of multifunctional transradial prostheses," IEEE Trans. neural Syst. Rehabil. Eng., vol. 18, no. 2, pp. $185-192,2010$.

[12] M. Zecca, S. Micera, M. C. Carrozza, and P. Dario, "Control of multifunctional prosthetic hands by processing the electromyographic signal," Crit. Rev. Biomed. Eng., vol. 30, no. 4-6, pp. 459-485, 2002.

[13] T. R. Farrell and R. F. Weir, "The optimal controller delay for myoelectric prostheses," IEEE Trans. neural Syst. Rehabil. Eng., vol. 15 , no. 1, pp. 111-118, 2007.

[14] G. R. Naik, A. H. Al-Timemy, and H. T. Nguyen, "Transradial Amputee Gesture Classification Using an Optimal Number of sEMG Sensors: An Approach Using ICA Clustering," IEEE Trans. Neural Syst. Rehabil. Eng., vol. 24, no. 8, pp. 837-846, 2016.

[15] R. N. Khushaba, S. Kodagoda, D. Liu, and G. Dissanayake, "Electromyogram (EMG) based fingers movement recognition using neighborhood preserving analysis with QR-decomposition," in 2011 Seventh International Conference on Intelligent Sensors, Sensor Networks and Information Processing, 2011, pp. 1-105.

[16] M. Atzori et al., "Effect of clinical parameters on the control of myoelectric robotic prosthetic hands," J. Rehabil. Res. Dev., vol. 53, no. 3, pp. 345-358, 2016.

[17] M. Atzori, M. Cognolato, and H. Müller, "Deep learning with convolutional neural networks applied to electromyography data: A resource for the classification of movements for prosthetic hands," Front. Neurorobot., vol. 10, no. 9, 2016.

[18] C. Cipriani, M. Controzzi, G. Kanitz, and R. Sassu, "The Effects of Weight and Inertia of the Prosthesis on the Sensitivity of Electromyographic Pattern Recognition in Relax State," JPO J. Prosthetics Orthot., vol. 24, no. 2, pp. 86-92, 2012.

[19] N. Jiang, S. Muceli, B. Graimann, and D. Farina, "Effect of arm position on the prediction of kinematics from EMG in amputees," Med. Biol. Eng. Comput., vol. 51, no. 1-2, pp. 143-151, 2013.

[20] E. Scheme, A. Fougner, Ø. Stavdahl, A. C. Chan, and K. Englehart, "Examining the adverse effects of limb position on pattern recognition based myoelectric control," in Engineering in Medicine and Biology Society (EMBC), 2010 Annual International Conference of the IEEE, 2010, pp. 6337-6340.

[21] C. J. De Luca, "Physiology and mathematics of myoelectric signals," IEEE Trans. Biomed. Eng., vol. 26, no. 6, pp. 313-325, Jun. 1979

[22] R. Merletti and P. A. Parker, Electromyography: Physiology, Engineering, and Non-Invasive Applications. John Wiley \& Sons, 2004

[23] A. M. Simon, L. J. Hargrove, B. A. Lock, and T. A. Kuiken, "A decision-based velocity ramp for minimizing the effect of misclassifications during real-time pattern recognition control," IEEE Trans. Biomed. Eng., vol. 58, no. 8, pp. 2360-2368, 2011.

[24] E. J. Scheme, B. S. Hudgins, and K. B. Englehart, "Confidence-based rejection for improved pattern recognition myoelectric control," IEEE Trans. Biomed. Eng., vol. 60, no. 6, pp. 1563-1570, 2013.

[25] “COAPT." [Online]. Available: https://coaptengineering.com/.

[26] B. Hudgins, P. Parker, and R. N. Scott, "A new strategy for multifunction myoelectric control," IEEE Trans. Biomed. Eng., vol. 40, no. 1, pp. 82-94, 1993.

[27] Y. Yamazaki, M. Suzuki, and T. Mano, "An electromyographic volley at initiation of rapid isometric contractions of the elbow," Brain Res. Bull., vol. 30, no. 1-2, pp. 181-187, 1993.

[28] K. Englehart, B. Hudgins, M. Stevenson, and P. A. Parker, "Classification of transient myoelectric signals using a dynamic feedforward neural network," in World Congress Neural Networks Proceedings, 1995.

[29] S. Leowinata, B. Hudgins, and P. A. Parker, "A multifunction myoelectric control strategy using an array of electrodes," in 16th Annual Congress of the International Society Electrophysiology and Kinesiology, 1998. 
[30] P. J. Gallant, “An Approach to Myoelectric Control Using a SelfOrganizing Neural Network for Feature Extraction," Queens University, Kingston, Ontario, Canada, 1993.

[31] N. Jiang, T. Lorrain, and D. Farina, "A state-based, proportional myoelectric control method: online validation and comparison with the clinical state-of-the-art,” J. Neuroeng. Rehabil., vol. 11, no. 1, p. 110, Jan. 2014.

[32] N. Hogan, E. Bizzi, F. A. Mussa-Ivaldi, and T. Flash, "Controlling multijoint motor behavior," Exerc. Sport Sci. Rev., vol. 15, pp. 153190, 1987.

[33] J. Z. Zheng, S. D. La Rosa, and A. M. Dollar, "An Investigation of Grasp Type and Frequency in Daily Household and Machine Shop Tasks," Proc. 2011 IEEE Int. Conf. Robot. Autom., pp. 4169-4175, 2011.

[34] J. T. Belter, J. L. Segil, A. M. Dollar, and R. F. ff. Weir, "Mechanical design and performance specifications of anthropomorphic prosthetic hands: a review," J. Rehabil. Res. Dev., vol. 50, no. 5, pp. 599-618, 2013.

[35] E. A. Clancy and K. A. Farry, "Adaptive whitening of the electromyogram to improve amplitude estimation," IEEE Trans. Biomed. Eng., vol. 47, no. 6, pp. 709-719, Jun. 2000.

[36] M. Jeannerod, "The timing of natural prehension movements," J. Mot. Behav., vol. 16, no. 3, pp. 235-254, 1984.

[37] K. Englehart, B. Hudgins, and A. D. C. Chan, "Continuous multifunction myoelectric control using pattern recognition," Technol. Disabil., vol. 15, no. 2, pp. 95-103, 2003.

[38] S. Solnik, P. Rider, K. Steinweg, P. Devita, and T. Hortobágyi, "TeagerKaiser energy operator signal conditioning improves EMG onset detection,” Eur. J. Appl. Physiol., vol. 110, no. 3, pp. 489-498, 2010.

[39] G. Staude, C. Flachenecker, M. Daumer, and W. Wolf, "Onset detection in surface electromyographic signals: A systematic comparison of methods," EURASIP J. Appl. Signal Processing, vol. 2001, no. 2, pp. 67-81, 2001.

[40] J. Liu, D. Ying, W. Z. Rymer, and P. Zhou, "Robust muscle activity onset detection using an unsupervised electromyogram learning framework," PLoS One, vol. 10, no. 6, p. e0127990, 2015.

[41] D. Yang, H. Zhang, Y. Gu, and H. Liu, "Accurate EMG onset detection in pathological, weak and noisy myoelectric signals," Biomed. Signal Process. Control, vol. 33, pp. 306-315, 2017.

[42] E. L. Allwein, R. E. Schapire, and Y. Singer, "Reducing multiclass to binary: A unifying approach for margin classifiers," J. Mach. Learn. Res., vol. 1, pp. 113-141, 2001.

[43] J. Fürnkranz, "Round Robin Classification," J. Mach. Learn. Res., vol. 2, pp. 721-747, 2002.

[44] A. Fougner, Ø. Stavdahl, P. J. Kyberd, Y. G. Losier, and P. A. Parker, "Control of Upper Limb Prostheses: Terminology and Proportional Myoelectric Control - A Review," IEEE Trans. Neural Syst. Rehabil. Eng., vol. 20, no. 5, pp. 663-677, 2012.

[45] M. Asghari Oskoei and H. Hu, "Myoelectric control systems-A survey," Biomed. Signal Process. Control, vol. 2, no. 4, pp. 275-294, Oct. 2007.

[46] M. Santello and J. F. Soechting, "Gradual molding of the hand to object contours," J. Neurophysiol., vol. 79, no. 3, pp. 1307-20, Mar. 1998.

[47] Y. Paulignan, C. MacKenzie, R. Marteniuk, and M. Jeannerod, "The coupling of arm and finger movements during prehension," Exp. Brain Res., vol. 79, no. 2, pp. 431-435, Sep. 1990.

[48] Y. Geng, P. Zhou, and G. Li, "Toward attenuating the impact of arm positions on electromyography pattern-recognition based motion classification in transradial amputees," J. Neuroeng. Rehabil., vol. 9, no. 1, pp. 1-11, 2012.

[49] A. Chadwell, L. P. J. Kenney, S. B. Thies, A. J. Galpin, and J. Head, "The reality of myoelectric prostheses: Understanding what makes these devices difficult for some users to control," Front. Neurorobot., vol. 10, no. 7, pp. 1-21, 2016.

[50] R. N. Khushaba, M. Takruri, J. V. Miro, and S. Kodagoda, "Towards limb position invariant myoelectric pattern recognition using timedependent spectral features," Neural networks, vol. 55, pp. 42-58, 2014.

[51] J. He, D. Zhang, N. Jiang, X. Sheng, D. Farina, and X. Zhu, "User adaptation in long-term, open-loop myoelectric training: implications for EMG pattern recognition in prosthesis control," J. Neural Eng., vol. 12, no. 4, p. 46005, 2015.

[52] T. Lorrain, N. Jiang, and D. Farina, "Influence of the training set on the accuracy of surface EMG classification in dynamic contractions for the control of multifunction prostheses," J. Neuroeng. Rehabil., vol. 8, no. 25, pp. 1-8, 2011. 